\title{
EULER-MARUYAMA APPROXIMATIONS IN \\ MEAN-REVERTING STOCHASTIC VOLATILITY \\ MODEL UNDER REGIME-SWITCHING
}

XUERONG MAO, AUBREY TRUMAN, AND CHENGGUI YUAN

Received 28 December 2005; Revised 9 February 2006; Accepted 9 February 2006

Stochastic differential equations (SDEs) under regime-switching have recently been developed to model various financial quantities. In general, SDEs under regime-switching have no explicit solutions, so numerical methods for approximations have become one of the powerful techniques in the valuation of financial quantities. In this paper, we will concentrate on the Euler-Maruyama (EM) scheme for the typical hybrid mean-reverting $\theta$-process. To overcome the mathematical difficulties arising from the regime-switching as well as the non-Lipschitz coefficients, several new techniques have been developed in this paper which should prove to be very useful in the numerical analysis of stochastic systems.

Copyright (c) 2006 Xuerong Mao et al. This is an open access article distributed under the Creative Commons Attribution License, which permits unrestricted use, distribution, and reproduction in any medium, provided the original work is properly cited.

\section{Introduction}

In the well-known Black-Scholes model, the asset price is described by a geometric Brownian motion

$$
d X(t)=\mu X(t) d t+v X(t) d w_{1}(t)
$$

where $w_{1}(t)$ is a scalar Brownian motion, $\mu$ is the rate of return of the underlying asset, and $\nu$ is the volatility. In this classical model, Black and Scholes [2] assumed that the rate of return and the volatility are constants. However, it has been proved by many authors (see, e.g., $[5,14,16,20]$ ) that the volatility is itself an Itô process in many situations. For instance, Hull and White [16] assume that the instantaneous variance $V=v^{2}$ obeys another geometric Brownian motion

$$
d V(t)=\alpha V(t) d t+\beta V(t) d w_{2}(t)
$$

where $\alpha, \beta$ are constants while $w_{2}(t)$ is another Brownian motion and $w_{1}(t)$ and $w_{2}(t)$ 
have correlation $\rho$. Heston [14] assumes that the variance $V$ obeys the mean-reverting square root process

$$
d V(t)=\alpha(\lambda-V(t)) d t+\beta \sqrt{V(t)} d w_{2}(t)
$$

while the mean-reverting process

$$
d V(t)=\alpha(\lambda-V(t)) d t+\beta V(t) d w_{2}(t)
$$

is also proposed as the volatility process by others. In particular, Lewis [18] proposes the mean-reverting $\theta$-process

$$
d V(t)=\alpha(\lambda-V(t)) d t+\beta V^{\theta}(t) d w_{2}(t)
$$

where $\theta \geq 1 / 2$. This process unifies processes (1.3) and (1.4).

On the other hand, the rate of return $\mu$ is not a constant either and there is a strong evidence to indicate that it is a Markov jump process (see, e.g., $[4,6,7,10,17,22,23,25]$ ). Of course, when the rate jumps, the volatility will jump accordingly. For example, the hybrid geometric Brownian motion

$$
d X(t)=\mu(r(t)) X(t) d t+v(r(t)) X(t) d w_{1}(t)
$$

has been proposed by several authors (see $[27,28]$ among others). Here, $r(t)$ is a Markov chain with a finite state space $\mathcal{M}=\{1,2, \ldots, N\}$ and $\mu, v$ are mappings from $\mathcal{M}$ to $[0, \infty)$. Equation (1.6) is also known as the geometric Brownian motion under regime-switching. We observe that in this model, the volatility is also assumed to obey a Markov jump process. Recalling the stochastic volatility models mentioned above, we may more reasonably assume that the volatility process obeys a stochastic differential equation (SDE) under regime-switching, for example, the hybrid mean-reverting $\theta$-process

$$
d V(t)=\alpha(r(t))(\lambda(r(t))-V(t)) d t+\beta(r(t)) V^{\theta}(t) d w_{2}(t) .
$$

Such stochastic models under regime-switching have recently been developed to model various financial quantities, for example, option pricing $[4,10-13,17]$, stock returns [6, $7,23]$, and portfolio optimization $[22,25]$. In particular, the mean-reverting square root process under regime-switching or, more generally, (1.7) has found its considerable use as a model for volatility and interest rate. In general, SDEs under regime-switching have no explicit solutions so the Monte Carlo simulations have become one of the powerful techniques in valuation of financial quantities, for example, option price (see $[9,15,24])$. However, there is currently a lack of theory that guarantees the convergence of the Monte Carlo simulations for SDEs under regime-switching in finance. This is due to the fact that most of SDEs under regime-switching in finance are nonlinear and non-Lipschitzian so we cannot appeal to the standard convergence theory for numerical simulations, as typified by [26], to deduce that the numerically computed paths are accurate for small step sizes.

In this paper, we will concentrate on the Euler-Maruyama (EM) scheme for the typical hybrid mean-reverting $\theta$-process (1.7) but the theory established here can certainly be developed to cope with other SDEs under regime-switching in finance. In Section 2, we will 
introduce necessary notations and investigate the global positive or nonnegative solutions to the mean-reverting $\theta$-process under regime-switching. The EM numerical scheme will be defined in Section 3, where we will explain how to simulate discrete Markov chains, and hence the EM approximate solutions. In Section 4, we will show that the EM solutions converge to the exact solution. The path-dependent option with the volatility described by the hybrid mean-reverting $\theta$-process will be discussed in Section 5, while Section 6 contains applications to other financial quantities.

\section{Nonnegative solutions}

Throughout this paper, we let $\left(\Omega, \mathscr{F}_{,},\left\{\mathscr{F}_{t}\right\}_{t \geq 0}, \mathbb{P}\right)$ be a complete probability space with a filtration $\left\{\mathscr{F}_{t}\right\}_{t \geq 0}$ satisfying the usual conditions (i.e., it is increasing and right continuous while $\mathscr{F}_{0}$ contains all $\mathbb{P}$-null sets). Let $w(t)$ be a scalar Brownian motion defined on the probability space. Let $|\cdot|$ denote the Euclidean norm. Let $r(t), t \geq 0$, be a rightcontinuous Markov chain on the probability space taking values in a finite state space $M=\{1,2, \ldots, N\}$ with the generator $\Gamma=\left(\gamma_{i j}\right)_{N \times N}$ given by

$$
\mathbb{P}\{r(t+\delta)=j \mid r(t)=i\}= \begin{cases}\gamma_{i j} \delta+o(\delta) & \text { if } i \neq j, \\ 1+\gamma_{i j} \delta+o(\delta) & \text { if } i=j,\end{cases}
$$

where $\delta>0$. Here $\gamma_{i j}$ is the transition rate from $i$ to $j$ and $\gamma_{i j}>0$ if $i \neq j$ while

$$
\gamma_{i i}=-\sum_{j \neq i} \gamma_{i j}
$$

We assume that the Markov chain $r(\cdot)$ is independent of the Brownian motion $w(\cdot)$. It is well known that almost every sample path of $r(\cdot)$ is a right-continuous step function with a finite number of sample jumps in any finite subinterval of $\mathbb{R}_{+}:=[0, \infty)$.

Let $1 / 2 \leq \theta \leq 1$. Consider the mean-reverting $\theta$-process under regime-switching of the form

$$
d S(t)=\lambda(r(t))[\mu(r(t))-S(t)] d t+\sigma(r(t)) S^{\theta}(t) d w(t), \quad t \geq 0,
$$

with initial data $S(0)=S_{0}>0$ and $r(0)=i_{0} \in M$. Here $\lambda(i), \mu(i), \sigma(i), i \in \mathcal{M}$, are positive constants. The initial data $S_{0}$ and $i_{0}$ could be random, but the Markov property ensures that it is sufficient to consider only the case when both $S_{0}$ and $i_{0}$ are constants. We note that the case when $\theta=1 / 2$ and the state space of the Markov chain $M=\{1\}$ corresponds to the classical mean-reverting square root process (1.3) (without regime-switching).

Since (2.3) is mainly used to model stochastic volatility or interest rate or an asset price, it is critical that the solution $S(t)$ will never become negative. The following lemma reveals this nonnegative property. 
4 EM approximation in mean-reverting regime-switching model

Lemma 2.1. For given any initial data $S(0)=S_{0}>0$ and $r(0)=i_{0} \in M$, the solution $S(t)$ of (2.3) will never become negative with probability 1.

Proof. Clearly, the statement of the lemma is equivalent to that the solution of equation

$$
d S(t)=\lambda(r(t))[\mu(r(t))-S(t)] d t+\sigma(r(t))|S(t)|^{\theta} d w(t), \quad t \geq 0,
$$

will never become negative with probability 1 for any initial data $S(0)=S_{0}>0$ and $r(0)=$ $i_{0} \in \mathcal{M}$. To show this, let $a_{0}=1$, and for each integer $k=1,2, \ldots$,

$$
a_{k}= \begin{cases}e^{-k(k+1)} & \text { if } \theta=\frac{1}{2}, \\ {\left[\frac{(2 \theta-1) k(k+1)}{2}\right]^{1 /(1-2 \theta)}} & \text { if } \frac{1}{2}<\theta \leq 1,\end{cases}
$$

so that

$$
\int_{a_{k}}^{a_{k-1}} \frac{d u}{u^{2 \theta}}=k
$$

For each $k=1,2, \ldots$, there clearly exists a continuous function $\psi_{k}(u)$ with support in $\left(a_{k}, a_{k-1}\right)$ such that

$$
0 \leq \psi_{k}(u) \leq \frac{2}{k u^{2 \theta}} \quad \text { for } a_{k}<u<a_{k-1}
$$

and $\int_{a_{k}}^{a_{k-1}} \psi_{k}(u) d u=1$. Define $\varphi_{k}(x)=0$ for $x \geq 0$ and

$$
\varphi_{k}(x)=\int_{0}^{-x} d y \int_{0}^{y} \psi_{k}(u) d u \quad \text { for } x<0
$$

Then $\varphi_{k} \in C^{2}(\mathbb{R}, \mathbb{R})$ and has the following properties:

(i) $-1 \leq \varphi_{k}^{\prime}(x) \leq 0$ for $a_{k}<x<a_{k-1}$, or otherwise $\varphi_{k}^{\prime}(x)=0$;

(ii) $\left|\varphi_{k}^{\prime \prime}(x)\right| \leq 2 / k|x|^{2 \theta}$ for $a_{k}<x<a_{k-1}$, or otherwise $\varphi_{k}^{\prime}(x)=0$;

(iii) $|x|-a_{k-1} \leq \varphi_{k}(x) \leq|x|$ for all $x \in \mathbb{R}$.

Let $\bar{\lambda}=\max _{i \in \mathcal{M}} \lambda(i)$, let $\bar{\mu}=\max _{i \in \mathcal{M}} \mu(i)$, and let $\bar{\sigma}=\max _{i \in \mathcal{M}} \sigma(i)$. Now for any $t \geq 0$, by the well-known Itô formula (see $[19,21]$ ), we can derive that

$$
\begin{aligned}
\mathbb{E} \varphi_{k}(S(t))= & \varphi_{k}\left(S_{0}\right) \\
& +\mathbb{E} \int_{0}^{t}\left[\lambda(r(u))(\mu(r(u))-S(u)) \varphi_{k}^{\prime}(S(u))+\frac{\sigma^{2}(r(u))}{2}|S(u)|^{2 \theta} \varphi_{k}^{\prime \prime}(S(r(u)))\right] d u \\
\leq & \frac{\bar{\sigma}^{2} t}{k} .
\end{aligned}
$$

Hence

$$
-a_{k-1} \leq \mathbb{E} S^{-}(t)-a_{k-1} \leq \frac{\bar{\sigma}^{2} t}{k}
$$


where $S^{-}(t)=-S(t)$ if $S(t)<0$, or otherwise $S^{-}(t)=0$. Letting $k \rightarrow \infty$, we get that $\mathbb{E} S^{-}(t)$ $=0$ for all $t \geq 0$. This implies that $S(t) \geq 0$ for all $t \geq 0$ with probability 1 as required. tion.

Furthermore, the following lemma reveals the (strictly) positive property of the solu-

Lemma 2.2. For given any initial data $S(0)=S_{0}>0$ and $r(0)=i_{0} \in M$, the solution $S(t)$ of (2.3) will remain positive with probability 1 , namely $S(t)>0$ for all $t \geq 0$ almost surely, if one of the following two conditions holds:

(i) $1 / 2<\theta \leq 1$;

(ii) $\theta=1 / 2$ and $\sigma^{2}(i) \leq 2 \lambda(i) \mu(i)$ for all $i \in M$.

To show this lemma, let us first invoke the standard results, for example, results of Gīhman and Skorohod [8], to establish the following result.

Lemma 2.3. Consider the mean-reverting $\theta$-process

$$
d X(t)=\lambda(\mu-X(t)) d t+\sigma X^{\theta}(t) d w(t)
$$

on $t \geq 0$ with initial value $X(0)=x_{0}>0$, where $1 / 2 \leq \theta \leq 1$ and $\lambda, \mu, \sigma$ are all positive constants. Then

(i) with probability 1 , the solution $X(t)$ takes an infinite time to reach origin 0 if either $1 / 2<\theta \leq 1$ or $\theta=1 / 2$ with $\sigma^{2} \leq 2 \lambda \mu$;

(ii) with positive probability, the solution $X(t)$ reaches the origin in finite time if $\theta=1 / 2$ and $\sigma^{2}>2 \lambda \mu$.

Proof. The coefficients of (2.11)

$$
a(z)=\lambda(\mu-z), \quad b(z)=\sigma z^{\theta}
$$

obey the linear growth condition on $z \in \mathbb{R}_{+}$, so the solution will never explode to infinity in any finite time with probability 1 . We therefore need only to consider if it reaches the origin in finite time or not.

Consider

$$
L_{1}=\int_{0}^{1} \exp \left\{-\int_{1}^{x} \frac{2 a(z)}{b^{2}(z)} d z\right\} d x
$$

When $1 / 2<\theta \leq 1$, this gives

$$
L_{1}=C_{1} \int_{0}^{1} \exp \left(-\frac{2 \lambda \mu x^{1-2 \theta}}{\sigma^{2}(1-2 \theta)}+\frac{2 \lambda x^{2-2 \theta}}{\sigma^{2}(2-2 \theta)}\right) d x,
$$

where $C_{1}$ is a positive constant. A simple inspection shows that $L_{1}$ diverges. Hence, the required result when $1 / 2<\theta \leq 1$ follows from Gihman and Skorohod [8, Chapter 21]. Similarly, when $\theta=1 / 2$,

$$
L_{1}=C_{2} \int_{0}^{1} \exp \left(-\frac{2 \lambda \mu}{\sigma^{2}} \log (x)\right) d x=C_{2} \int_{0}^{1} x^{-2 \lambda \mu / \sigma^{2}} d x
$$


where $C_{2}$ is another positive constant. It is then easy to see that $L_{1}=\infty$ if $2 \lambda \mu \geq \sigma^{2}$ while $L_{1}<\infty$ if $2 \lambda \mu<\sigma^{2}$. The required results corresponding to $2 \lambda \mu \geq \sigma^{2}$ or $2 \lambda \mu<\sigma^{2}$ when $\theta=1 / 2$ follow from Gīhman and Skorohod [8, Chapter 21] again.

Using Lemma 2.3, we can now prove Lemma 2.2.

Proof of Lemma 2.2. It is well known (see, e.g., [1]) that there is a sequence of stopping times $0=\tau_{0}<\tau_{1}<\cdots<\tau_{k} \rightarrow \infty$ such that the Markov chain $r(t)$ has the representation

$$
r(t)=\sum_{k=0}^{\infty} r\left(\tau_{k}\right) I_{\left[\tau_{k}, \tau_{k+1}\right)}(t), \quad t \geq 0,
$$

where $I_{A}$ is the indicator function of set $A$. Hence, for $t \in\left[0, \tau_{1}\right],(2.3)$ becomes

$$
d S(t)=\lambda\left(r_{0}\right)\left[\mu\left(r_{0}\right)-S(t)\right] d t+\sigma\left(r_{0}\right) S^{\theta}(t) d w(t)
$$

with $S(0)>0$. This is a mean-reverting $\theta$-process of type (2.11). Applying Lemma 2.3, we observe that $S(t)>0$ for all $t \in\left[0, \tau_{1}\right]$ with probability 1 under the conditions of Lemma 2.2. Now, for $t \in\left[\tau_{1}, \tau_{2}\right]$, (2.3) becomes

$$
d S(t)=\lambda\left(r\left(\tau_{1}\right)\right)\left[\mu\left(r\left(\tau_{1}\right)\right)-S(t)\right] d t+\sigma\left(r\left(\tau_{1}\right)\right) S^{\theta}(t) d w(t)
$$

with initial value $S\left(\tau_{1}\right)>0$ a.s. Again, this is a mean-reverting $\theta$-process of type (2.11). By Lemma 2.3, we see that $S(t)>0$ for all $t \in\left[\tau_{1}, \tau_{2}\right]$ with probability 1 . Repeating this procedure, we see that $S(t)>0$ for all $t \geq 0$ with probability 1 as required.

It is still remaining open whether the solution $S(t)$ will reach the origin in finite time with positive probability in the case when $\theta=1 / 2$ while $\sigma^{2}(i) \leq 2 \lambda(i) \mu(i)$ does not hold for all $i \in \mathcal{M}$.

\section{The Euler-Maruyama method}

To define the Euler-Maruyama approximate solution, we will need the following lemma (see [1]).

Lemma 3.1. Given $\Delta>0$, let $r_{k}^{\Delta}=r(k \Delta)$ for $k=0,1,2, \ldots$ Then $\left\{r_{k}^{\Delta}, k=0,1,2, \ldots\right\}$ is a discrete-time Markov chain with the one-step transition probability matrix

$$
P(\Delta)=\left(P_{i j}(\Delta)\right)_{N \times N}=e^{\Delta \Gamma}
$$

Given a step size $\Delta>0$, the discrete-time Markov chain $\left\{r_{k}^{\Delta}, k=0,1,2, \ldots\right\}$ can be simulated as follows: compute the one-step transition-probability matrix

$$
P(\Delta)=\left(P_{i j}(\Delta)\right)_{N \times N}=e^{\Delta \Gamma}
$$


Let $r_{0}^{\Delta}=i_{0}$ and generate a random number $\xi_{1}$ which is uniformly distributed in $[0,1]$. Define

$$
r_{1}^{\Delta}= \begin{cases}i_{1} \quad & \text { if } i_{1} \in \mathcal{M}-\{N\} \text { is such that } \sum_{j=1}^{i_{1}-1} P_{i_{0}, j}(\Delta) \leq \xi_{1}<\sum_{j=1}^{i_{1}} P_{i_{0}, j}(\Delta), \\ N & \text { if } \sum_{j=1}^{N-1} P_{i_{0}, j}(\Delta) \leq \xi_{1},\end{cases}
$$

where we set $\sum_{i=1}^{0} P_{i_{0}, j}(\Delta)=0$ as usual. Generate independently a new random number $\xi_{2}$ which is again uniformly distributed in $[0,1]$ and then define

$$
r_{2}^{\Delta}= \begin{cases}i_{2} \quad \text { if } i_{2} \in \mathcal{M}-\{N\} \text { is such that } \sum_{j=1}^{i_{2}-1} P_{r_{1}^{\Delta}, j}(\Delta) \leq \xi_{2}<\sum_{j=1}^{i_{2}} P_{r_{1}^{\Delta}, j}(\Delta), \\ N \quad \text { if } \sum_{j=1}^{N-1} P_{r_{1}^{\Delta}, j}(\Delta) \leq \xi_{2} .\end{cases}
$$

Repeating this procedure, a trajectory of $\left\{r_{k}^{\Delta}, k=0,1,2, \ldots\right\}$ can be generated. This procedure can be carried out independently to obtain more trajectories.

After explaining how to simulate the discrete-time Markov chain $\left\{r_{k}^{\Delta}, k=0,1, \ldots\right\}$, we can now define the EM approximate solution for (2.3). Given a step size $\Delta>0$, let $t_{k}=k \Delta$ for $k=0,1,2, \ldots$. Compute the discrete approximations $s_{k} \approx S\left(t_{k}\right)$ by setting $s_{0}=S_{0}, r_{0}^{\Delta}=$ $i_{0}$ and forming

$$
s_{k+1}=s_{k}+\lambda\left(r_{k}^{\Delta}\right)\left(\mu\left(r_{k}^{\Delta}\right)-s_{k}\right) \Delta+\sigma\left(r_{k}^{\Delta}\right)\left|s_{k}\right|^{\theta} \Delta w_{k}, \quad k=0,1,2, \ldots,
$$

where $\Delta w_{k}=w\left(t_{k+1}\right)-w\left(t_{k}\right)$. Let

$$
\bar{s}(t)=s_{k}, \quad \bar{r}(t)=r_{k}^{\Delta} \quad \text { for } t \in\left[t_{k}, t_{k+1}\right), k=0,1,2, \ldots,
$$

and define the continuous EM approximate solution by

$$
s(t)=s_{0}+\int_{0}^{t} \lambda(\bar{r}(u))[\mu(\bar{r}(u))-\bar{s}(u)] d u+\int_{0}^{t} \sigma(\bar{r}(u))|\bar{s}(u)|^{\theta} d w(u) .
$$

Note that $s\left(t_{k}\right)=\bar{s}\left(t_{k}\right)=s_{k}$, that is, $s(t)$ and $\bar{s}(t)$ coincide with the discrete approximate solution at the grid points.

\section{Convergence of the EM approximate solution}

Since the coefficients of (2.3) satisfy the linear growth condition, by [26], we have the following lemma.

Lemma 4.1. Let $S(t)$ be the solution of (2.3). Then for any $p \geq 1$, there is a constant $K$, which is dependent on only $p, T, S_{0}$ but independent of $\Delta$, such that the exact solution and the EM 
8 EM approximation in mean-reverting regime-switching model

approximate solution to (2.3) have the property that

$$
\mathbb{E}\left[\sup _{0 \leq t \leq T}|S(t)|^{p}\right] \vee \mathbb{E}\left[\sup _{0 \leq t \leq T}|s(t)|^{p}\right] \leq K
$$

From this follows easily the following useful result.

LEMma 4.2. There is a constant $C$, which is independent of $\Delta$, such that

$$
\mathbb{E}|s(t)-\bar{s}(t)|^{2 \theta} \leq C \Delta^{\theta}, \quad \forall t \in[0, T]
$$

Proof. From now on, $C$ used in the proofs below will be a generic positive number independent of $\Delta$ but may have different values where it appears.

For any $t \in[0, T]$, let $k_{t}=[t / \Delta]$, the integer part of $t / \Delta$. By Lemma 4.1, we then derive that

$$
\mathbb{E}|\bar{s}(t)-s(t)|^{2} \leq 4(\bar{\lambda} \vee \bar{\lambda} \bar{\mu} \vee \bar{\sigma}) \mathbb{E}\left[\left(1+\left|s_{k_{t}}\right|^{2}\right)\left(\Delta^{2}+\left|w(t)-w\left(k_{t} \Delta\right)\right|^{2}\right)\right] \leq C \Delta .
$$

So, since $1 / 2 \leq \theta \leq 1$, by the Lyapunov inequality, we get

$$
\mathbb{E}|\bar{s}(t)-s(t)|^{2 \theta} \leq\left[\mathbb{E}|\bar{s}(t)-s(t)|^{2}\right]^{\theta} \leq C \Delta^{\theta}
$$

as required.

We can now state one of our main results.

Theorem 4.3. For each integer $k=1,2, \ldots$,

$$
\sup _{0 \leq t \leq T} \mathbb{E}|S(t)-s(t)| \leq e^{\bar{\lambda} T}\left[e^{-k(k-1) / 2}+\frac{4 \bar{\sigma}^{2} T}{k}+\left(\frac{1}{k a_{k}^{2 \theta}}+1\right)\left(C \Delta^{\theta}+o(\Delta)\right)\right]
$$

where $C$ is a constant which is independent of the step size $\Delta$ and $\bar{\lambda}, \bar{\sigma}$ have been defined in the proof of Lemma 2.1.

Proof. Note that

$$
\begin{aligned}
S(t)-s(t)= & \int_{0}^{t}[\lambda(r(u)) \mu(r(u))-\lambda(\bar{r}(u)) \mu(\bar{r}(u))-\lambda(r(u)) S(u)+\lambda(\bar{r}(u)) \bar{s}(u)] d u \\
& +\int_{0}^{t}\left[\sigma(r(u))|S(u)|^{\theta}-\sigma(\bar{r}(u))|\bar{s}(u)|^{\theta}\right] d w(u) .
\end{aligned}
$$


Let $\varphi_{k}$ be the same as defined in the proof of Lemma 2.1. Applying the Itô formula gives $\mathbb{E} \varphi_{k}(S(t)-s(t))$

$$
\begin{gathered}
=\mathbb{E} \int_{0}^{t} \varphi_{k}^{\prime}(S(u)-s(u))[\lambda(r(u)) \mu(r(u))-\lambda(\bar{r}(u)) \mu(\bar{r}(u)) \\
-\lambda(r(u)) S(u)+\lambda(\bar{r}(u)) \bar{s}(u)] d u \\
+\frac{1}{2} \mathbb{E} \int_{0}^{t} \varphi_{k}^{\prime \prime}(S(u)-s(u))\left[\sigma(r(u))|S(u)|^{\theta}-\sigma(\bar{r}(u))|\bar{s}(u)|^{\theta}\right]^{2} d u=: I(t)+\frac{1}{2} J(t) .
\end{gathered}
$$

By property (i) of $\varphi_{k}$,

$$
\begin{aligned}
|I(t)| \leq & \mathbb{E} \int_{0}^{t} \mid \varphi_{k}^{\prime}(S(u)-s(u))[\lambda(r(u)) \mu(r(u))-\lambda(\bar{r}(u)) \mu(\bar{r}(u)) \\
& -\lambda(r(u)) S(u)+\lambda(\bar{r}(u)) \bar{s}(u)] \mid d u \\
\leq & \mathbb{E} \int_{0}^{t}|\lambda(r(u)) \mu(r(u))-\lambda(\bar{r}(u)) \mu(\bar{r}(u))| d u \\
& +\mathbb{E} \int_{0}^{t}|\lambda(r(u)) S(u)-\lambda(\bar{r}(u)) \bar{s}(u)| d u .
\end{aligned}
$$

Let $n=[T / \Delta]$, the integer part of $T / \Delta$. Then

$$
\begin{aligned}
& \mathbb{E} \int_{0}^{T} \mid \lambda(r(u)) \mu(r(u))-\lambda(\bar{r}(u)) \mu(\bar{r}(u)) \mid d u \\
& \quad=\sum_{k=0}^{n} \mathbb{E} \int_{t_{k}}^{t_{k+1}}|\lambda(r(u)) \mu(r(u))-\lambda(\bar{r}(u)) \mu(\bar{r}(u))| d u
\end{aligned}
$$

with $t_{n+1}$ being now set to be $T$. Let $I_{G}$ be the indicator function of set $G$ and compute

$$
\begin{aligned}
\mathbb{E} \int_{t_{k}}^{t_{k+1}} & |\lambda(r(u)) \mu(r(u))-\lambda(\bar{r}(u)) \mu(\bar{r}(u))| d u \leq 2 \bar{\lambda} \bar{\mu} E \int_{t_{k}}^{t_{k+1}} I_{\left\{r(u) \neq r\left(t_{k}\right)\right\}} d u \\
\leq & 2 \bar{\lambda} \bar{\mu} \int_{t_{k}}^{t_{k+1}} \mathbb{P}\left(r(u) \neq r\left(t_{k}\right)\right) d u=2 \bar{\lambda} \bar{\mu} \int_{t_{k}}^{t_{k+1}} \sum_{i \in \mathcal{M}} \mathbb{P}\left(r\left(t_{k}\right)=i\right) \mathbb{P}\left(r(u) \neq i \mid r\left(t_{k}\right)=i\right) d u \\
\quad & 2 \bar{\lambda} \bar{\mu} \int_{t_{k}}^{t_{k+1}} \sum_{i \in \mathcal{M}} \mathbb{P}\left(r\left(t_{k}\right)=i\right) \sum_{j \neq i}\left(\gamma_{i j}\left(u-t_{k}\right)+o\left(u-T_{k}\right)\right) \\
\leq & 2 \bar{\lambda} \bar{\mu}\left[\max _{1 \leq i \leq N}\left(-\gamma_{i i}\right) \Delta+o(\Delta)\right] \Delta .
\end{aligned}
$$


Therefore,

$$
\mathbb{E} \int_{0}^{T}|\lambda(r(u)) \mu(r(u))-\lambda(\bar{r}(u)) \mu(\bar{r}(u))| d u \leq 2 \bar{\lambda} \bar{\mu}\left[\max _{1 \leq i \leq N}\left(-\gamma_{i i}\right) \Delta+o(\Delta)\right] .
$$

On the other hand,

$$
\begin{aligned}
& \mathbb{E} \int_{0}^{t}|\lambda(r(u)) S(u)-\lambda(\bar{r}(u)) \bar{s}(u)| d u \\
& \quad \leq \mathbb{E} \int_{0}^{t}|\lambda(r(u))-\lambda(\bar{r}(u))||\bar{s}(u)| d u+\mathbb{E} \int_{0}^{t} \lambda(r(u))|S(u)-\bar{s}(u)| d u
\end{aligned}
$$

But

$$
\begin{aligned}
\mathbb{E} \int_{0}^{t} \mid & \lambda(r(u))-\lambda(\bar{r}(u))|| \bar{s}(u) \mid d u \\
\quad= & \sum_{k=0}^{n} \int_{t_{k}}^{t_{k+1}} \mathbb{E}\left[\mathbb{E}\left[\left|\lambda(r(u))-\lambda\left(r\left(t_{k}\right)\right)\right|\left|s_{k}\right| \mid I_{\left\{r(u) \neq r\left(t_{k}\right)\right\}}\right]\right] d u \\
\quad= & \sum_{k=0}^{n} \int_{t_{k}}^{t_{k+1}} \mathbb{E}\left[\mathbb{E}\left[\left|\lambda(r(u))-\lambda\left(r\left(t_{k}\right)\right)\right| \mid I_{\left\{r(u) \neq r\left(t_{k}\right)\right\}}\right] \mathbb{E}\left[\left|s_{k}\right| \mid I_{\left\{r(u) \neq r\left(t_{k}\right)\right\}}\right]\right],
\end{aligned}
$$

where in the last step we use the fact that $s_{k}$ and $I_{\left\{r(u) \neq r\left(t_{k}\right)\right\}}$ are conditionally independent with respect to the $\sigma$-algebra generated by $r\left(t_{k}\right)$. In the same way as in $(4.10)$, we have

$$
\begin{aligned}
& \mathbb{E} \int_{0}^{t} \mid \lambda(r(u))-\lambda(\bar{r}(u))|| \bar{s}(u) \mid d u \\
& \leq 2 \bar{\lambda} \bar{\mu}\left[\max _{1 \leq i \leq N}\left(-\gamma_{i i}\right) \Delta+o(\Delta)\right] \int_{0}^{T} \mathbb{E}|\bar{s}(u)| d u .
\end{aligned}
$$

So, by Lemma 4.1,

$$
\mathbb{E} \int_{0}^{t}|\lambda(r(u))-\lambda(\bar{r}(u)) \bar{s}(u)| d u \leq 2(1+K) \bar{\lambda} \bar{\mu}\left[\max _{1 \leq i \leq N}\left(-\gamma_{i i}\right) \Delta+o(\Delta)\right]
$$

Substituting (4.15) into (4.12) and using Lemma 4.2, we obtain

$$
\begin{aligned}
& \mathbb{E} \int_{0}^{t} \mid \lambda(r(u)) S(u)-\lambda(\bar{r}(u)) \bar{s}(u) \mid d u \\
& \quad \leq C \Delta+o(\Delta)+\bar{\lambda} \mathbb{E} \int_{0}^{t}|S(u)-\bar{s}(u)| d u \\
& \quad \leq C \Delta+o(\Delta)+\bar{\lambda} \mathbb{E} \int_{0}^{t}|S(u)-s(u)| d u,
\end{aligned}
$$


where $C$ is a positive constant independent of $\Delta$ and it may change line by line. This, together with (4.11), yields

$$
|I(t)| \leq C \Delta+o(\Delta)+\bar{\lambda} \mathbb{E} \int_{0}^{t}|S(u)-s(u)| d u .
$$

In the following, we will estimate $J(t)$ :

$$
\begin{aligned}
|J(t)| \leq & 2 \bar{\sigma}^{2} \mathbb{E} \int_{0}^{t}\left|\varphi_{k}^{\prime \prime}(S(u)-s(u))\right|\left(|S(u)|^{\theta}-|\bar{s}(u)|^{\theta}\right)^{2} d u \\
& +2 \mathbb{E} \int_{0}^{t}\left|\varphi_{k}^{\prime \prime}(S(u)-s(u))\right|[\sigma(r(u))-\sigma(\bar{r}(u))]^{2}|S(u)|^{2 \theta} d u .
\end{aligned}
$$

Using property (ii) of $\varphi_{k}$ and Lemma 4.2, we have

$$
\begin{aligned}
\mathbb{E} \int_{0}^{t} \mid & \varphi_{k}^{\prime \prime}(S(u)-s(u)) \mid\left(|S(u)|^{\theta}-|\bar{s}(u)|^{\theta}\right)^{2} d u \\
\leq & \mathbb{E} \int_{0}^{t}\left|\varphi_{k}^{\prime \prime}(S(u)-s(u))\right||S(u)-\bar{s}(u)|^{2 \theta} d u \\
\leq & 2^{2 \theta-1} \mathbb{E} \int_{0}^{t}\left|\varphi_{k}^{\prime \prime}(S(u)-s(u))\right||S(u)-s(u)|^{2 \theta} d u \\
& +2^{2 \theta-1} \mathbb{E} \int_{0}^{t}\left|\varphi_{k}^{\prime \prime}(S(u)-s(u))\right||s(u)-\bar{s}(u)|^{2 \theta} d u \\
\leq & 2 \mathbb{E} \int_{0}^{t} \frac{2}{k} I_{\left\{a_{k}<|S(u)-s(u)|<a_{k-1}\right\}} d u+2 \int_{0}^{t} \frac{2}{k a_{k}^{2 \theta}} \mathbb{E}|s(u)-\bar{s}(u)|^{2 \theta} d u \leq \frac{4 T}{k}+\frac{C \Delta^{\theta}}{k a_{k}^{2 \theta}} .
\end{aligned}
$$

In the same way as (4.15) was proved, we can show that

$$
\begin{aligned}
& \mathbb{E} \int_{0}^{t}\left|\varphi_{k}^{\prime \prime}(S(u)-s(u))\right|[\sigma(r(u))-\sigma(\bar{r}(u))]^{2}|S(u)|^{2 \theta} d u \\
& \quad \leq \mathbb{E} \int_{0}^{t} \frac{2}{k a_{k}^{2 \theta}}[\sigma(r(u))-\sigma(\bar{r}(u))]^{2}|S(u)|^{2 \theta} d u \leq \frac{C \Delta+o(\Delta)}{k a_{k}^{2 \theta}} .
\end{aligned}
$$

Substituting (4.20) and (4.19) into (4.18), we have

$$
|J(t)| \leq \frac{8 \bar{\sigma}^{2} T}{k}+\frac{C \Delta^{\theta}+o(\Delta)}{k a_{k}^{2 \theta}} .
$$

Therefore,

$$
\mathbb{E} \varphi_{k}(S(t)-s(t)) \leq \frac{4 \bar{\sigma}^{2} T}{k}+\frac{C \Delta^{\theta}+o(\Delta)}{k a_{k}^{2 \theta}}+C \Delta+o(\Delta)+2 \bar{\lambda} \mathbb{E} \int_{0}^{t}|S(u)-s(u)| d u .
$$


12 EM approximation in mean-reverting regime-switching model

Noting that

$$
\mathbb{E} \varphi_{k}(S(t)-s(t)) \geq \mathbb{E}|S(t)-s(t)|-a_{k-1}
$$

gives

$$
\mathbb{E}|S(t)-s(t)| \leq a_{k-1}+\frac{4 \bar{\sigma}^{2} T}{k}+\left[\frac{1}{k a_{k}^{2 \theta}}+1\right]\left(C \Delta^{\theta}+o(\Delta)\right)+\bar{\lambda} \int_{0}^{t} \mathbb{E}|S(u)-s(u)| d u .
$$

The required assertion follows finally from the Gronwall inequality.

Next, we derive a bound for a stronger form of the error. This version uses an $L^{2}$ distance and places the supremum over time inside the expectation operation. The result below involves the $L^{1}$-error which is explicitly bounded in Theorem 4.3 , and hence is also computable.

Theorem 4.4. One has

$$
\mathbb{E}\left[\sup _{0 \leq t \leq T}(S(t)-s(t))^{2}\right] \leq e^{\left(8 \bar{\sigma}^{2}+2 \bar{\lambda}^{2}\right) T^{2}}\left(C \Delta+o(\Delta)+8 \bar{\sigma}^{2} T \sup _{0 \leq u \leq T} \mathbb{E}|S(u)-s(u)|\right) .
$$

Proof. For any $0 \leq t \leq T$, using the Cauchy-Schwarz inequality, we have

$$
\begin{aligned}
(S(t)- & s(t))^{2} \\
\leq & T \int_{0}^{t}[\lambda(r(u)) \mu(r(u))-\lambda(\bar{r}(u)) \mu(\bar{r}(u))-\lambda(r(u)) S(u)+\lambda(\bar{r}(u)) \bar{s}(u)]^{2} d u \\
& +\left(\int_{0}^{t}\left[\sigma(r(u))|S(u)|^{\theta}-\sigma(\bar{r}(u))|\bar{s}(u)|^{\theta}\right] d w(u)\right)^{2} .
\end{aligned}
$$

In the same way as (4.11) and (4.16) were proved, we derive

$$
\begin{aligned}
\mathbb{E} \int_{0}^{t}[ & \lambda(r(u)) \mu(r(u))-\lambda(\bar{r}(u)) \mu(\bar{r}(u))-\lambda(r(u)) S(u)+\lambda(\bar{r}(u)) \bar{s}(u)]^{2} d u \\
\leq & 2 \mathbb{E} \int_{0}^{t}[\lambda(r(u)) \mu(r(u))-\lambda(\bar{r}(u)) \mu(\bar{r}(u))]^{2} d u \\
& +\mathbb{E} \int_{0}^{t}[\lambda(r(u)) S(u)-\lambda(\bar{r}(u)) \bar{s}(u)]^{2} d u \\
\leq & C \Delta+o(\Delta)+2 \bar{\lambda}^{2} \mathbb{E} \int_{0}^{t}(S(u)-s(u))^{2} d u .
\end{aligned}
$$


Using the Doob martingale inequality (see [19]), we find that for any $t_{1} \in[0, T]$,

$$
\begin{aligned}
& \mathbb{E}\left[\sup _{0 \leq t \leq t_{1}}\left(\int_{0}^{t}\left[\sigma(r(u))|S(u)|^{\theta}-\sigma(\bar{r}(u))|\bar{s}(u)|^{\theta}\right] d w(u)\right)^{2}\right] \\
& \quad \leq 4 \mathbb{E} \int_{0}^{t_{1}}\left[\sigma(r(u))|S(u)|^{\theta}-\sigma(\bar{r}(u))|\bar{s}(u)|^{\theta}\right]^{2} d u \\
& \leq C \Delta+o(\Delta)+8 \bar{\sigma}^{2} \mathbb{E} \int_{0}^{t_{1}}|S(u)-s(u)|^{2 \theta} d u \\
& \leq C \Delta+o(\Delta)+8 \bar{\sigma}^{2} \mathbb{E} \int_{0}^{t_{1}}|S(u)-s(u)| d u+8 \bar{\sigma}^{2} \mathbb{E} \int_{0}^{t_{1}}|S(u)-s(u)|^{2} d u .
\end{aligned}
$$

Therefore,

$$
\begin{aligned}
\mathbb{E}\left[\sup _{0 \leq t \leq t_{1}}(S(t)-s(t))^{2}\right] \\
\leq C \Delta+o(\Delta)+\left(8 \bar{\sigma}^{2}+2 \bar{\lambda}^{2}\right) \mathbb{E} \int_{0}^{t_{1}}(S(u)-s(u))^{2} d u+8 \bar{\sigma}^{2} \mathbb{E} \int_{0}^{t_{1}}|S(u)-s(u)| d u \\
\leq C \Delta+o(\Delta)+\left(8 \bar{\sigma}^{2}+2 \bar{\lambda}^{2}\right) \int_{0}^{t_{1}} \mathbb{E}\left[\sup _{0 \leq u \leq v}(S(u)-s(u))^{2}\right] d v \\
\quad+8 \bar{\sigma}^{2} T \sup _{0 \leq u \leq T} \mathbb{E}|S(u)-s(u)| .
\end{aligned}
$$

An application of the Gronwall inequality completes the proof.

\section{Options under stochastic volatility and regime-switching}

In this section, we study the Heston stochastic volatility model under regime-switching, namely

$$
\begin{aligned}
& d X(t)=\lambda_{1}(r(t))\left[\mu_{1}(r(t))-X(t)\right] d t+\sigma_{1}(r(t)) X(t) \sqrt{V(t)} d w_{1}(t), \\
& d V(t)=\lambda_{2}(r(t))\left[\mu_{2}(r(t))-V(t)\right] d t+\sigma_{2}(r(t)) V^{\theta}(t) d w_{2}(t), \quad 0 \leq t \leq T .
\end{aligned}
$$

Here $V(t)$ is the volatility that feeds into the asset price $X(t)$. The Brownian motions $w_{1}(t)$ and $w_{2}(t)$ may be correlated. Naturally, we assume that the initial values $X(0)$ and $V(0)$ are both positive constants. Moreover, $\lambda_{1}, \sigma_{1}$, and so forth are all mappings from $\mathcal{M}$ to $\mathbb{R}_{+}$.

We begin with a lemma showing that the positivity in the initial data leads to the positive solution $X(t)$.

LEMma 5.1. If $V(t), t \in[0, T]$, is given by (5.2), then

$$
\mathbb{P}(X(t)>0 \forall 0 \leq t \leq T)=1 .
$$


Proof. By the well-known variation-of-constants formula (see, e.g., [19, Theorem 3.1, page 96$]), X(t)$ can be expressed explicitly as

$$
X(t)=\Psi(t)\left(X(0)+\int_{0}^{t} \frac{\lambda_{1}(r(s)) \mu_{1}(r(s))}{\Psi(s)} d s\right)
$$

where

$$
\Psi(t)=\exp \left(\int_{0}^{t}\left[-\lambda_{1}(r(s))-\frac{1}{2} \sigma_{1}^{2}(r(s)) V(s)\right] d s+\int_{0}^{t} \sigma_{1}(r(s)) \sqrt{V(s)} d w_{1}(s)\right)
$$

The assertion follows clearly.

Applying the EM method to (5.2) gives

$$
v_{k+1}=v_{k}+\lambda_{1}\left(r_{k}^{\Delta}\right)\left(\mu_{1}\left(r_{k}^{\Delta}\right)-v_{k}\right) \Delta+\sigma_{1}\left(r_{k}^{\Delta}\right)\left|v_{k}\right|^{\theta} \Delta w_{2, k}
$$

where $\Delta w_{2, k}=w_{2}\left(t_{k+1}\right)-w_{2}\left(t_{k}\right)$, while applying the EM method to (5.1) gives

$$
x_{k+1}=x_{k}+\lambda_{2}\left(r_{k}^{\Delta}\right)\left(\mu_{2}\left(r_{k}^{\Delta}\right)-x_{k}\right) \Delta+\sigma_{2}\left(r_{k}^{\Delta}\right) x_{k} \sqrt{\left|v_{k}\right|} \Delta w_{1, k}
$$

where $\Delta w_{1, k}=w_{1}\left(t_{k+1}\right)-w_{1}\left(t_{k}\right)$.

Let

$$
\bar{x}(t)=x_{k}, \quad \bar{v}(t)=v_{k} \quad \bar{r}(t)=r_{k}^{\Delta} \quad \text { for } t \in\left[t_{k}, t_{k+1}\right), k=0,1,2, \ldots,
$$

and define the continuous EM approximate solution by

$$
\begin{gathered}
x(t)=x_{0}+\int_{0}^{t} \lambda_{1}(\bar{r}(u))\left[\mu_{1}(\bar{r}(u))-\bar{x}(u)\right] d u+\int_{0}^{t} \sigma_{1}(\bar{r}(u)) \bar{x}(u) \sqrt{|\bar{v}(u)|} d w_{1}(u), \\
v(t)=v_{0}+\int_{0}^{t} \lambda_{2}(\bar{r}(u))\left[\mu_{2}(\bar{r}(u))-\bar{v}(u)\right] d u+\int_{0}^{t} \sigma_{2}(\bar{r}(u))|\bar{v}(u)|^{\theta} d w_{2}(u) .
\end{gathered}
$$

In the following, we will prove the result of strong convergence of the asset price $X(t)$. Lemma 5.2. For any given pair of positive numbers $p$ and $q$, define the stopping time

$$
\tau_{p q}=\inf \{t \geq 0: X(t)>p \text { or }|v(t)|>q\}
$$

Then

$$
\lim _{\Delta \rightarrow 0} \mathbb{E}\left(\sup _{0 \leq t \leq T}\left|X\left(t \wedge \tau_{p q}\right)-x\left(t \wedge \tau_{p q}\right)\right|^{2}\right)=0
$$


Proof. Fix $p$ and $q$ arbitrarily and write $\tau_{p q}=\tau$. For any $0 \leq t_{1} \leq T$,

$$
\begin{aligned}
X\left(t_{1} \wedge\right. & \tau)-x\left(t_{1} \wedge \tau\right) \\
= & \int_{0}^{t_{1} \wedge \tau}\left[\lambda_{1}(r(u)) \mu_{1}(r(u))-\lambda_{1}(\bar{r}(u)) \mu_{1}(\bar{r}(u))\right] d u \\
& -\int_{0}^{t_{1} \wedge \tau}\left[\lambda_{1}(r(u)) X(u)-\lambda_{1}(\bar{r}(u)) \bar{x}(u)\right] d u \\
& +\int_{0}^{t_{1} \wedge \tau}\left[\sigma_{1}(r(u)) X(u) \sqrt{V(u)}-\sigma_{1}(\bar{r}(u)) \bar{x}(u) \sqrt{|\bar{v}(u)|}\right] d w_{1}(u) \\
= & \int_{0}^{t_{1} \wedge \tau}\left[\lambda_{1}(r(u)) \mu_{1}(r(u))-\lambda_{1}(\bar{r}(u)) \mu_{1}(\bar{r}(u))\right] d u \\
& -\int_{0}^{t_{1} \wedge \tau} X(u)\left[\lambda_{1}(r(u))-\lambda_{1}(\bar{r}(u))\right] d u-\int_{0}^{t_{1} \wedge T} \lambda_{1}(\bar{r}(u))[X(u)-\bar{x}(u)] d u \\
& +\int_{0}^{t_{1} \wedge \tau} X(u) \sqrt{V(u)}\left[\sigma_{1}(r(u))-\sigma_{1}(\bar{r}(u))\right] d w_{1}(u) \\
& +\int_{0}^{t_{1} \wedge \tau} \sigma_{1}(\bar{r}(u)) \sqrt{\bar{v}(u)}[X(u)-\bar{x}(u)] d w_{1}(u) \\
& +\int_{0}^{t_{1} \wedge \tau} \sigma_{1}(\bar{r}(u)) X(u)[\sqrt{V(u)}-\sqrt{|\bar{v}(u)|}] d w_{1}(u) .
\end{aligned}
$$

By the Hölder inequality and the Doob martingale inequality, we have

$$
\begin{aligned}
\mathbb{E}\left(\sup _{0 \leq t_{1} \leq t}|X(t \wedge \tau)-x(t \wedge \tau)|^{2}\right) \\
\leq 32 t \mathbb{E} \int_{0}^{t \wedge \tau}\left[\lambda_{1}(r(u)) \mu_{1}(r(u))-\lambda_{1}(\bar{r}(u)) \mu_{1}(\bar{r}(u))\right]^{2} d u \\
+32 t \mathbb{E} \int_{0}^{t \wedge \tau} X^{2}(u)\left[\lambda_{1}(r(u))-\lambda_{1}(\bar{r}(u))\right]^{2} d u \\
+32 t \mathbb{E} \int_{0}^{t \wedge \tau} \lambda_{1}^{2}(\bar{r}(u))[X(u)-\bar{x}(u)]^{2} d u \\
+128 \mathbb{E} \int_{0}^{t \wedge \tau}(X(u) \sqrt{V(u)})^{2}\left[\sigma_{1}(r(u))-\sigma_{1}(\bar{r}(u))\right]^{2} d u \\
+128 \mathbb{E} \int_{0}^{t \wedge \tau}\left(\sigma_{1}(\bar{r}(u)) \sqrt{\bar{v}(u)}\right)^{2}[X(u)-\bar{x}(u)]^{2} d u \\
+128 \mathbb{E} \int_{0}^{t \wedge \tau}\left(\sigma_{1}(\bar{r}(u)) X(u)\right)^{2}[\sqrt{V(u)}-\sqrt{|\bar{v}(u)|}]^{2} d u
\end{aligned}
$$


Using Lemmas 4.1 and 4.2, the definition of $\tau$, and the techniques of the proof of (4.15), we derive that

$$
\begin{aligned}
& \mathbb{E}\left(\sup _{0 \leq t_{1} \leq t}\left|X\left(t_{1} \wedge \tau\right)-x\left(t_{1} \wedge \tau\right)\right|^{2}\right) \\
& \quad \leq 32\left[t+p^{2} t+4 p^{2} K^{2}+4 \bar{\sigma}_{1}^{2} p^{2}\right](C \Delta+o(\Delta))+32\left[t \bar{\lambda}_{1}^{2}+4 \bar{s}_{1} q\right] \mathbb{E} \int_{0}^{t \wedge \tau}[X(u)-\bar{x}(u)]^{2} d u \\
& \quad \leq C_{1} \Delta+o(\Delta)+C_{2} \mathbb{E} \int_{0}^{t \wedge \tau}[X(u)-\bar{x}(u)]^{2} d u \\
& \quad \leq C_{1} \Delta+o(\Delta)+C_{2} \mathbb{E} \int_{0}^{t \wedge \tau}[X(u)-x(u)]^{2} d u+C_{2} \mathbb{E} \int_{0}^{t \wedge \tau}[x(u)-\bar{x}(u)]^{2} d u
\end{aligned}
$$

where $C_{1}, C_{2}$, and the following $C_{3}$, and so forth are positive constants which may change line by line. For $0 \leq u \leq t \wedge \tau$, let $[u / \Delta]$ be the integer part of $u / \Delta$. Then

$x(u)-\bar{x}(u)=\int_{[u / \Delta] \Delta}^{u} \lambda_{1}(\bar{r}(u))\left[\mu_{1}(\bar{r}(u))-\bar{x}(u)\right] d u+\int_{[u / \Delta] \Delta}^{u} \sigma_{1}(\bar{r}(u)) \bar{x}(u) \sqrt{|\bar{v}(u)|} d w_{1}(u)$,

which yields

$$
|x(u)-\bar{x}(u)|^{2} \leq 4 \bar{\lambda}_{1}^{2}\left(\bar{\mu}_{1}^{2}+p^{2}\right) \Delta^{2}+2 \bar{\sigma}_{1}^{2} p^{2} q\left(w_{1}(u)-w_{1}\left(\left[\frac{u}{\Delta}\right] \Delta\right)\right)^{2} .
$$

Therefore

$$
\mathbb{E} \int_{0}^{t \wedge \tau}[x(u)-\bar{x}(u)]^{2} d u \leq C_{3} \Delta .
$$

By (5.15) and (5.18), we have

$$
\begin{aligned}
& \mathbb{E}\left(\sup _{0 \leq t_{1} \leq t}\left|X\left(t_{1} \wedge \tau\right)-x\left(t_{1} \wedge \tau\right)\right|^{2}\right) \\
& \quad \leq C_{4} \Delta+o(\Delta)+C_{5} \int_{0}^{t \wedge \tau} \mathbb{E}\left(\sup _{0 \leq t_{1} \leq u}\left|X\left(t_{1}\right)-x\left(t_{1}\right)\right|^{2}\right) d u .
\end{aligned}
$$

By the well-known Gronwall inequality,

$$
\mathbb{E}\left(\sup _{0 \leq t_{1} \leq t}\left|X\left(t_{1} \wedge \tau\right)-x\left(t_{1} \wedge \tau\right)\right|^{2}\right) \leq\left[C_{4} \Delta+o(\Delta)\right] e^{C_{5} T} .
$$

The required assertion (5.12) follows by letting $\Delta \rightarrow 0$.

LEMMA 5.3. The continuous EM approximate solution (5.10) obeys

$$
\mathbb{E}\left(\sup _{0 \leq t \leq T}|v(t)|\right) \leq\left(1+2 v_{0}+2 \bar{\lambda}_{2} \bar{\mu}_{2} T\right) e^{\left(2 \bar{\lambda}_{2}+9 \bar{\sigma}_{2}^{2}\right) T} \quad \forall T>0
$$

where $\bar{\lambda}_{2}=\max _{i \in \mathcal{M}} \lambda_{2}(i)$ and $\bar{\mu}_{2}$ and $\bar{\sigma}_{2}$ are defined similarly. 
Proof. By the well-known Burkholder-Davis-Gunday inequality, we derive from (5.10) that for $0 \leq t \leq T$,

$$
\begin{aligned}
\mathbb{E}\left(\sup _{0 \leq t_{1} \leq t}\left|v\left(t_{1}\right)\right|\right) \leq & v_{0}+\int_{0}^{t_{1}}\left|\lambda_{2}(\bar{r}(u))\left[\mu_{2}(\bar{r}(u))-\bar{v}(u)\right]\right| d u \\
& +3 \mathbb{E}\left[\int_{0}^{t}\left(\sigma_{2}(\bar{r}(u))|\bar{v}(u)|^{\theta}\right)^{2} d u\right]^{1 / 2} \\
\leq & v_{0}+\bar{\lambda}_{2} \bar{\mu}_{2} T+\bar{\lambda}_{2} \int_{0}^{t} \mathbb{E}|\bar{v}(u)| d u \\
& +3 \bar{\sigma}_{2} \mathbb{E}\left[\left(\sup _{0 \leq t_{1} \leq t}\left|v\left(t_{1}\right)\right|^{2 \theta-1}\right) \int_{0}^{t}|\bar{v}(u)| d u\right]^{1 / 2} \\
\leq & v_{0}+\bar{\lambda}_{2} \bar{\mu}_{2} T+\bar{\lambda}_{2} \int_{0}^{t} \mathbb{E}|\bar{v}(u)| d u \\
& +\frac{1}{2} \mathbb{E}\left(\sup _{0 \leq t_{1} \leq t}\left|v\left(t_{1}\right)\right|^{2 \theta-1}\right)+\frac{9}{2} \bar{\sigma}_{2}^{2} \mathbb{E} \int_{0}^{t}|\bar{v}(u)| d u \\
\leq & v_{0}+\bar{\lambda}_{2} \bar{\mu}_{2} T+\frac{1}{2}\left[1+\mathbb{E}\left(\sup _{0 \leq t_{1} \leq t}\left|v\left(t_{1}\right)\right|\right)\right] \\
& +\left(\bar{\lambda}_{2}+\frac{9}{2} \bar{\sigma}_{2}^{2}\right) \int_{0}^{t} \mathbb{E}\left(\sup _{0 \leq t_{1} \leq u}\left|v\left(t_{1}\right)\right|\right) d u .
\end{aligned}
$$

This yields

$$
\mathbb{E}\left(\sup _{0 \leq t_{1} \leq t}\left|v\left(t_{1}\right)\right|\right) \leq 1+2 v_{0}+2 \bar{\lambda}_{2} \bar{\mu}_{2} T+\left(2 \bar{\lambda}_{2}+9 \bar{\sigma}_{2}^{2}\right) \int_{0}^{t} \mathbb{E}\left(\sup _{0 \leq t_{1} \leq u}\left|v\left(t_{1}\right)\right|\right) d u .
$$

An application of the Gronwall inequality implies assertion (5.21).

Broadie and Kaya [3] have recently shown how to simulate exactly the solution to the Heston model without regime-switching when the volatility process is described by the mean-reverting square root process (1.3). This is due to the closed form of the solution to the mean-reverting square root process (1.3). However, there is so far no closed form for the solution of the mean-reverting $\theta$-process (1.5) if $1 / 2<\theta \leq 1$, not mentioning the hybrid mean-reverting $\theta$-process (2.3). However, the EM method established above provides us with a numerical scheme to carry out the Monte Carlo simulation for the option price if the underlying asset price follows the Heston model under regime-switching (5.1) and (5.2).

Let $K$ be the exercise price. Define the payoff for the European put option

$$
\mathscr{P}=\mathbb{E}\left[(K-X(T))^{+}\right] .
$$

Accordingly, the payoff based on the numerical method (5.8) is

$$
\mathscr{P}_{\Delta}=\mathbb{E}\left[(K-\bar{x}(T))^{+}\right]
$$


Theorem 5.4. In the notation above,

$$
\lim _{\Delta \rightarrow 0}\left|\mathscr{P}_{\Delta}-\mathscr{P}\right|=0
$$

Furthermore, let us consider the more complicated barrier option which is a pathdependent option. Let $K$ be the exercise price and let $b$ be a barrier. For the Heston model under regime-switching (5.1) and (5.2), the payoff for the barrier European put option is given by

$$
U=\mathbb{E}\left[(X(T)-K)^{+} I_{\{0 \leq X(t) \leq b, 0 \leq t \leq T\}}\right]
$$

while the payoff based on the numerical method (5.8) is

$$
U_{\Delta}=\mathbb{E}\left[(\bar{x}(T)-K)^{+} I_{\{0 \leq \bar{x}(t) \leq b, 0 \leq t \leq T\}}\right] .
$$

Theorem 5.5. In the notation above,

$$
\lim _{\Delta \rightarrow 0}\left|U_{\Delta}-U\right|=0
$$

Theorem 5.4 and Theorem 5.5 can be proved in the same way as in [15] using Lemmas 5.1-5.3 as well as the strong convergence results established in the previous sections since the proofs presented in [15] depend only on the strong convergence properties and the conclusions of Lemmas 5.1-5.3 rather than the specified form of the underlying equations. It is in this spirit that we see that our results can be applied to compute other financial quantities numerically as described in the following sections.

\section{Further applications to finance}

In this section, we assume that the underlying asset price obeys the mean-reverting $\theta$ process under regime-switching, namely (2.3). We will omit the proofs, since they are similar to the proofs without regime-switching presented by [15].

6.1. Bonds. In the case where $S(t)$ in (2.3) models the short-term interest rate dynamics, the price of a bond is given by

$$
B(0, T)=\mathbb{E}\left[\exp ^{\left(-\int_{0}^{T} S(t) d t\right)}\right] .
$$

Using the step function $\bar{s}(t)$ in (3.6), a natural approximation to $B(0, T)$ is

$$
\overline{B(0, T)}=\mathbb{E}\left[\exp ^{\left(-\int_{0}^{T}|\bar{s}(t)| d t\right)}\right] .
$$

For this approximation, we have the following result.

Theorem 6.1. One has

$$
\lim _{\Delta \rightarrow 0}|\overline{B(0, T)}-B(0, T)|=0
$$


6.2. Single barrier option. We now consider a single barrier option, which, at expiry time $T$, pays the European value if $S(t)$ never exceeded the fixed barrier $b$, and pays zero otherwise. We suppose that the expected payoff is computed from a Monte Carlo simulation based on (3.5). The following theorem shows that the expected payoff from the numerical method will converge to the exact expected payoff as $\Delta \rightarrow 0$.

Theorem 6.2. Let $S(t)$ and $\bar{s}(t)$ be defined by (2.3) and (3.6), respectively. Let $K$ be the exercise price and let $b$ be a barrier. Define

$$
\begin{gathered}
V=\mathbb{E}\left[(S(T)-K)^{+} I_{\{0 \leq S(t) \leq b, 0 \leq t \leq T\}}\right], \\
V_{\Delta}=\mathbb{E}\left[(\bar{s}(T)-K)^{+} I_{\{0 \leq \bar{s}(t) \leq b, 0 \leq t \leq T\}}\right] .
\end{gathered}
$$

Then

$$
\lim _{\Delta \rightarrow 0}\left|V_{\Delta}-V\right|=0
$$

\section{Acknowledgments}

The authors would like to thank the referees for helpful comments and suggestions. The authors would also like to thank the financial supports from the Engineering and Physical Sciences Research Council (EPSRC) and the Royal Society.

\section{References}

[1] W. J. Anderson, Continuous-Time Markov Chains, Springer Series in Statistics: Probability and Its Applications, Springer, New York, 1991.

[2] F. Black and M. Scholes, The pricing of options and corporate liabilities, Journal of Political Economy 81 (1973), 637-659.

[3] M. Broadie and Kaya, Exact simulation of stochastic volatility and other affine jump diffusion processes, Working paper, 2003.

[4] J. Buffington and R. J. Elliott, American options with regime switching, International Journal of Theoretical and Applied Finance 5 (2002), no. 5, 497-514.

[5] J. C. Cox, J. E. Ingersoll Jr., and S. A. Ross, A theory of the term structure of interest rates, Econometrica 53 (1985), no. 2, 385-407.

[6] A. David, Fluctuating confidence in stock markets: Implications for returns and volatility, Journal of Financial and Quantitative Analysis 32 (1997), 427-462.

[7] J. B. Detemple, Further results on asset pricing with incomplete information, Journal of Economic Dynamics \& Control 15 (1991), no. 3, 425-453.

[8] I. İ. Gîhman and A. V. Skorohod, Stochastic Differential Equations, Ergebnisse der Mathematik und ihrer Grenzgebiete, vol. 72, Springer, New York, 1972.

[9] P. Glasserman, Monte Carlo Methods in Financial Engineering, Applications of Mathematics (New York), vol. 53, Springer, New York, 2004.

[10] X. Guo, An explicit solution to an optimal stopping problem with regime switching, Journal of Applied Probability 38 (2001), no. 2, 464-481.

[11] _ Information and option pricings, Quantitative Finance 1 (2001), no. 1, 38-44.

[12] X. Guo and L. Shepp, Some optimal stopping problems with nontrivial boundaries for pricing exotic options, Journal of Applied Probability 38 (2001), no. 3, 647-658.

[13] X. Guo and Q. Zhang, Closed-form solutions for perpetual American put options with regime switching, SIAM Journal on Applied Mathematics 64 (2004), no. 6, 2034-2049. 
[14] S. L. Heston, A closed-form solution for options with stochastic volatility with applications to bond and currency options, Review of Financial Studies 6 (1993), no. 2, 327-343.

[15] D. J. Higham and X. Mao, Convergence of Monte Carlo simulations involving the mean-reverting square root process, Journal of Computational Finance 8 (2005), 35-61.

[16] J. Hull and A. White, The pricing of options on assets with stochastic volatilities, Journal of Finance 42 (1987), 281-300.

[17] A. Jobert and L. C. G. Rogers, Option pricing with Markov-modulated dynamics, preprint, 2004.

[18] A. L. Lewis, Option Valuation under Stochastic Volatility. With Mathematica Code, Finance Press, California, 2000.

[19] X. Mao, Stochastic Differential Equations and Their Applications, Horwood Publishing Series in Mathematics \& Applications, Horwood, Chichester, 1997.

[20] R. C. Merton, Theory of rational option pricing, Bell Journal of Economics and Management Science 4 (1973), 141-183.

[21] A. V. Skorokhod, Asymptotic Methods in the Theory of Stochastic Differential Equations, Translations of Mathematical Monographs, vol. 78, American Mathematical Society, Rhode Island, 1989.

[22] R. H. Stockbridge, Portfolio optimization in markets having stochastic rates, Stochastic Theory and Control (Lawrence, KS, 2001), Lecture Notes in Control and Information Sciencs, vol. 280, Springer, Berlin, 2002, pp. 447-458.

[23] P. Veronesi, Stock market overreactions to bad news in good times: a rational expectations equilibrium model, Review of Financial Studies 12 (1999), no. 5, 975-1007.

[24] S. Wu and Y. Zeng, A general equilibrium model of the term structure of interest rates under regimeswitching risk, International Journal of Theoretical and Applied Finance 8 (2005), no. 7, 839869.

[25] G. Yin and X. Y. Zhou, Markowitz's mean-variance portfolio selection with regime switching: from discrete-time models to their continuous-time limits, IEEE Transactions on Automatic Control 49 (2004), no. 3, 349-360.

[26] C. Yuan and X. Mao, Convergence of the Euler-Maruyama method for stochastic differential equations with Markovian switching, Mathematics and Computers in Simulation 64 (2004), no. 2, 223-235.

[27] Q. Zhang, Stock trading: an optimal selling rule, SIAM Journal on Control and Optimization 40 (2001), no. 1, 64-87.

[28] Q. Zhang and G. Yin, On nearly optimal controls of hybrid LQG problems, IEEE Transactions on Automatic Control 44 (1999), no. 12, 2271-2282.

Xuerong Mao: Department of Statistics and Modelling Science, University of Strathclyde, Glasgow G1 1XH, Scotland, UK

E-mail address: xuerong@stams.strath.ac.uk

Aubrey Truman: Department of Mathematics, School of Physical Sciences, University of Wales Swansea, Swansea SA2 8PP, Wales, UK

E-mail address: a.truman@swansea.ac.uk

Chenggui Yuan: Department of Mathematics, School of Physical Sciences,

University of Wales Swansea, Swansea SA2 8PP, Wales, UK

E-mail address: c.yuan@swansea.ac.uk 


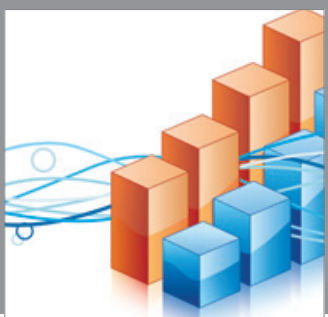

Advances in

Operations Research

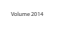

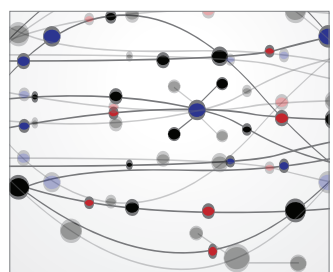

\section{The Scientific} World Journal
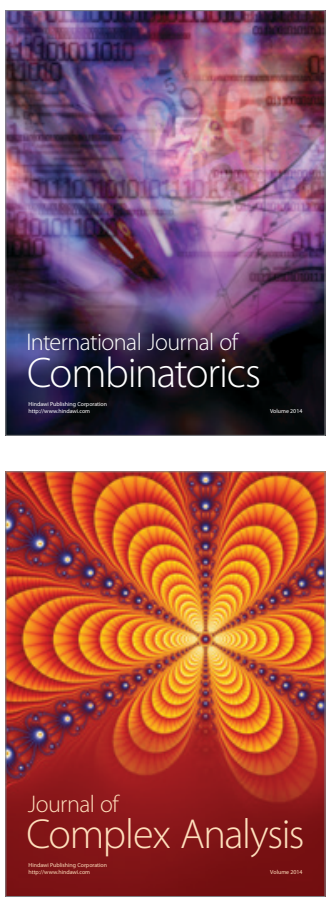

International Journal of

Mathematics and

Mathematical

Sciences
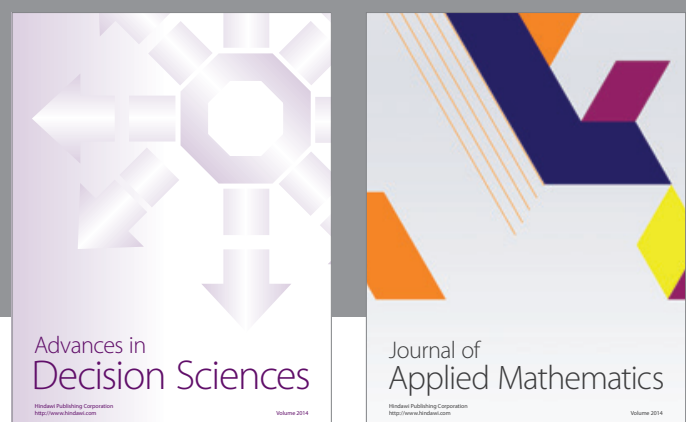

Journal of

Applied Mathematics
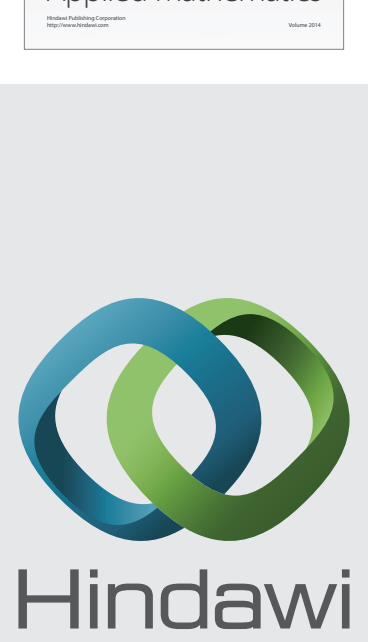

Submit your manuscripts at http://www.hindawi.com
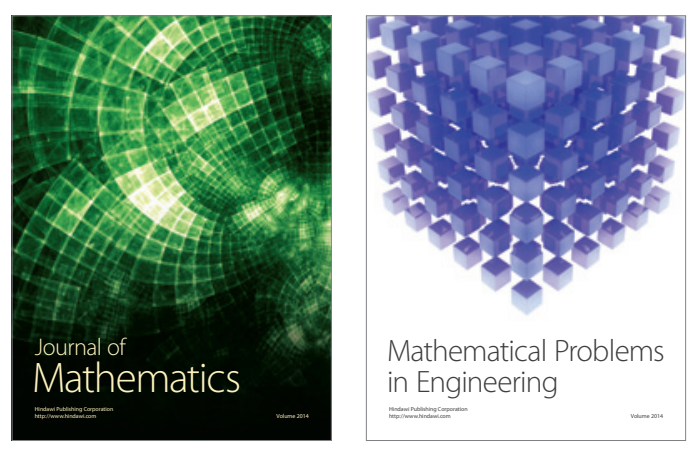

Mathematical Problems in Engineering
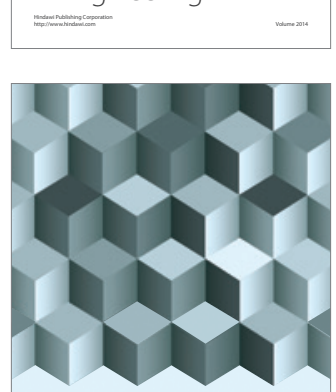

Journal of

Function Spaces
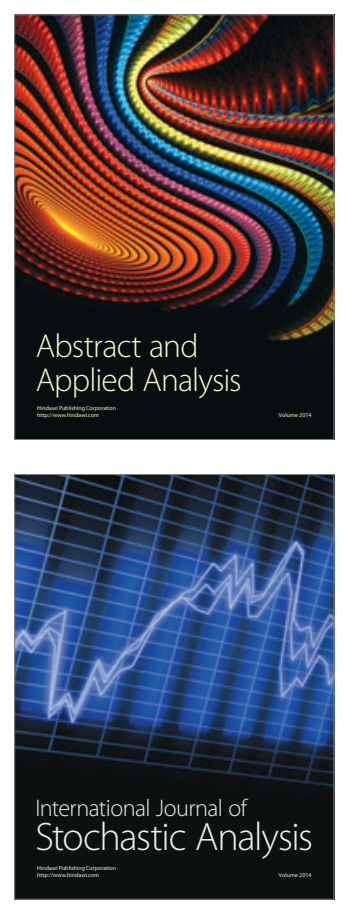

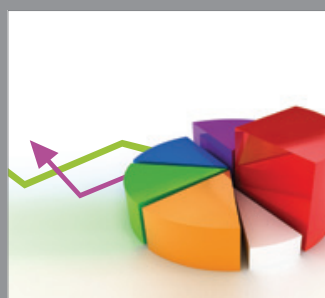

ournal of

Probability and Statistics

Promensencen
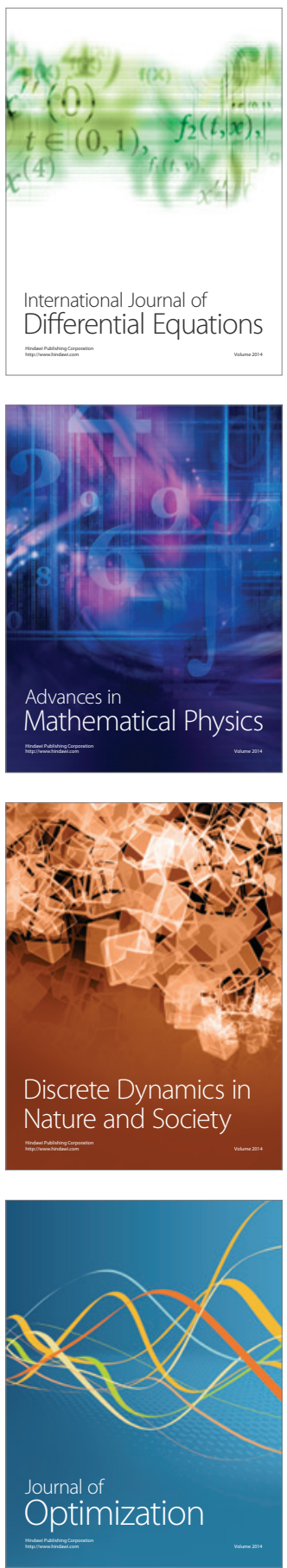